- and it is particularly irresponsible when the interventions are provided at public expense. On the other hand, we must avoid an innovation policy that cuts off new interventions prematurely. Some interventions that are not cost-effective at first may prove to be so over time and with greater experience in implement- ing them. It is in gathering this experience that the private part of the "should" question becomes important. Given a substantial market for untried, cutting-edge interventions that are not subsidized by the public purse, some innovations may prove to be costeffective in the long run. Such innovations should then be in- cluded in the publicly financed benefit package.

Disclosure forms provided by the author are available with the full text of this article at NEJM.org.

From Stanford University, Stanford, CA.

This article (10.1056/NEJMp1000200) was published on March 10, 2010, at NEJM.org.

Copyright (C) 2010 Massachusetts Medical Society.

\title{
AIDS in America - Forgotten but Not Gone
}

Wafaa M. El-Sadr, M.D., M.P.H., Kenneth H. Mayer, M.D., and Sally L. Hodder, M.D.

Over ver the past decade, limited attention has been paid to the human immunodeficiency virus (HIV) epidemic in the United States. The global epidemic particularly the epidemic in subSaharan Africa, where approximately two thirds of the world's population living with AIDS resides - has rightfully received most of the focus. Meanwhile, however, the prevalence of HIV infection within some U.S. populations now rivals that in some sub-Saharan African countries (see graph). For example, more than 1 in 30 adults in Washington, D.C., are HIV-infected - a prevalence higher than that reported in Ethiopia, Nigeria, or Rwanda. ${ }^{1}$ Certain U.S. subpopulations are particularly hard hit. In New York City, 1 in 40 blacks, 1 in 10 men who have sex with men, and 1 in 8 injection-drug users are HIV-infected, as are 1 in 16 black men in Washington, D.C. ${ }^{2}$ In several U.S. urban areas, the HIV prevalence among men who have sex with men is as high as $30 \%^{3}$ - as compared with a general-population prevalence of $7.8 \%$ in Kenya and $16.9 \%$ in South Africa.

During the first two decades of the epidemic, remarkable advances in preventing mother-tochild transmission, screening of blood and blood products, and behavior change among men who have sex with men resulted in significant decreases in new HIV infections in the United States - from approximately 130,000 in 1984 to about 60,000 in 1991. For the past decade, however, progress has been stalled. It had been anticipated that effective antiretroviral therapy, with its suppressive effect on viral replication, would reduce the overall rate of new infections, but this expectation has not been realized. More than half a million Americans became infected with HIV in the past decade, including about 56,000 in the past year. ${ }^{4}$ It is estimated that there are now more than 1 million HIV-infected Americans, more than $20 \%$ of whom are unaware of their infection.

Unlike the generalized HIV epidemics in sub-Saharan Africa, the U.S. epidemic primarily affects certain discrete geographic areas - especially urban areas of the Northeast and West Coast and cities and small towns in the South (see U.S. map). Within these areas, specific neighborhoods are often disproportionately affected (see New York City map), in part because of residents' engagement in unprotected sex within relatively insular socialsexual networks. Many of the populations most affected tend to have limited social mobility; thus, partner selection tends to concentrate transmission patterns and amplify spread within defined geographic areas.

Traditionally, researchers and policymakers concerned with HIV acquisition have concentrated on specific high-risk transmission behaviors, including injection-drug use, sex with multiple partners, and failure to use protective measures such as condoms or safe injection practices. It is now evident that among men who have sex with men, the use of drugs such as crystal methamphetamine - especially at sex parties and in venues such as bathhouses has contributed to risky behavior and HIV acquisition. Other disinhibiting substances, including alcohol and cocaine, are also associated with increased risk taking in these populations.

However, the extent of the risk of acquiring HIV in the United 


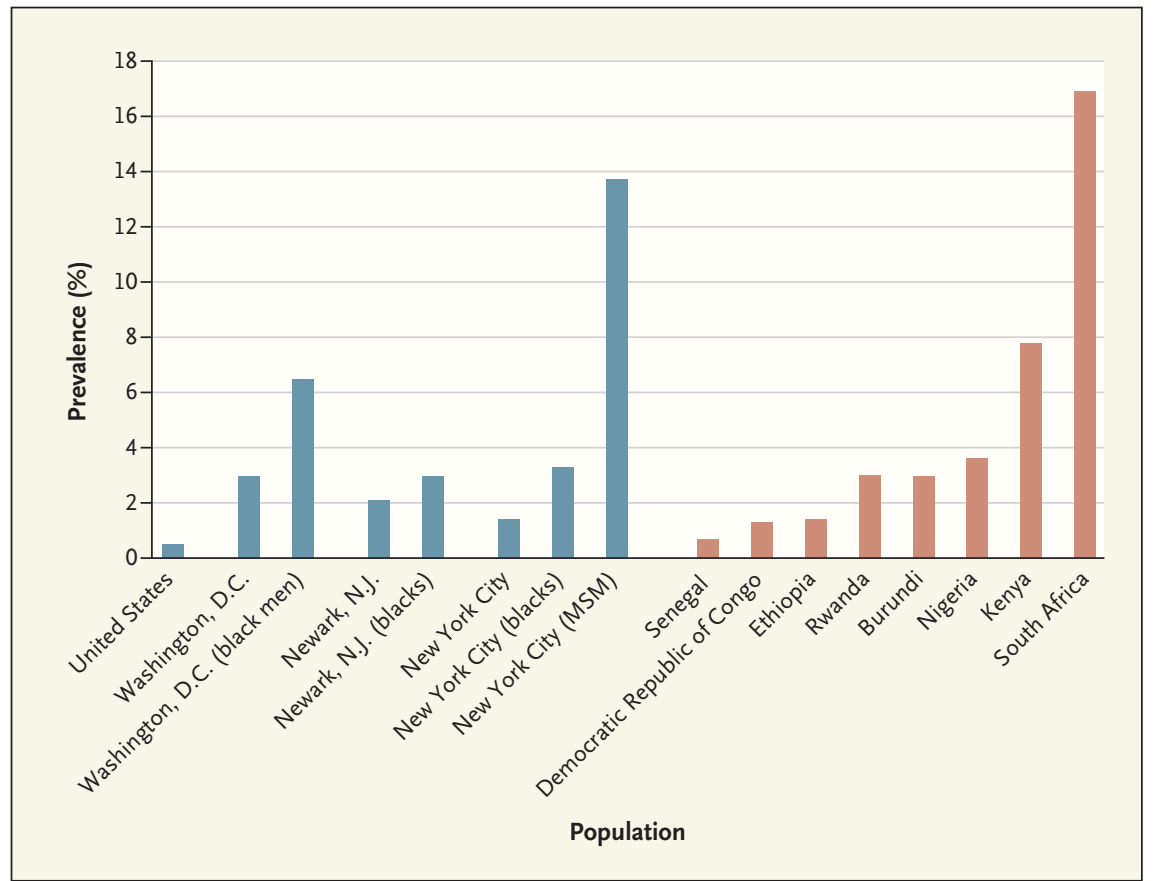

HIV Prevalence in Adults from Selected Countries in Sub-Saharan Africa and Subpopulations in the United States.

Data are from the Centers for Disease Control and Prevention, the District of Columbia Department of Health, the New Jersey Department of Health, the New York City Department of Health and Mental Hygiene, and the Joint United Nations Program on HIV/AIDS (UNAIDS). MSM denotes men who have sex with men.

States today is largely defined by a person's sexual network rather than his or her individual behaviors. Understanding the context and settings in which risk is increased may lead to more robust and effective preventive interventions. For example, black men who have sex with men are at increased risk for HIV infection in part because of its high prevalence in their sexual networks and their likelihood of choosing racially similar partners; they have also been shown to be less likely than their white counterparts to be aware of their HIV status and thus are more likely to unknowingly transmit HIV. ${ }^{5}$ Moreover, even those who are aware of their HIV infection may be less engaged in HIV care and less likely to avail themselves of antiretroviral therapy - behavior that lim- its the potential benefit of such therapy as a preventive strategy.

The situation is similar for black and Hispanic women, whose increased risk of HIV acquisition is attributable in greater part to their vulnerable social and economic situations and their sexual networks than to their own risky behaviors. Socioeconomic disadvantage and instability of partnerships due to high rates of incarceration among men in their communities may lead women to engage in concurrent relationships or serial monogamy. In addition, they may be unaware of their partners' HIV status or may be involved in abusive or economically dependent relationships and thus be unable to negotiate safer sex with their partners.

The specific characteristics of the U.S. HIV epidemic - low prevalence in the general population, high prevalence among the disenfranchised and socially marginalized, with a concentration in geographic hotspots - in combination with the various structural impediments to prevention create unique challenges for the design and implementation of effective interventions. Thus, a nuanced and targeted approach that avoids stigmatization of these populations is necessary. Structural interventions might include tackling the disproportionate incarceration of black and Hispanic men, urging health insurers to reimburse providers for preventive care, and using microcredit to help women out of poverty so that they avoid the perceived need to engage in commercial sex or other coercive sex.

Research tailored to specific populations is required if we are to gain the understanding needed to move forward. For example, how do we identify those people in the United States who are at greatest risk for HIV acquisition, especially among women? Although more than a quarter of new HIV infections in the United States occur in women (predominantly black or Hispanic women), identifying such at-risk women to engage them in prevention studies has proven particularly challenging. Research is also needed to identify interventions that will persuade men who have sex with men to undergo HIV testing, facilitate their disclosure of their HIV status to sexual partners, and promote negotiations for safer sexual practices; such interventions need to be implemented in the settings where such men may meet (e.g., in bars or on the Internet). Additional qualitative research is needed to understand how the targeted community uses various sources of information 


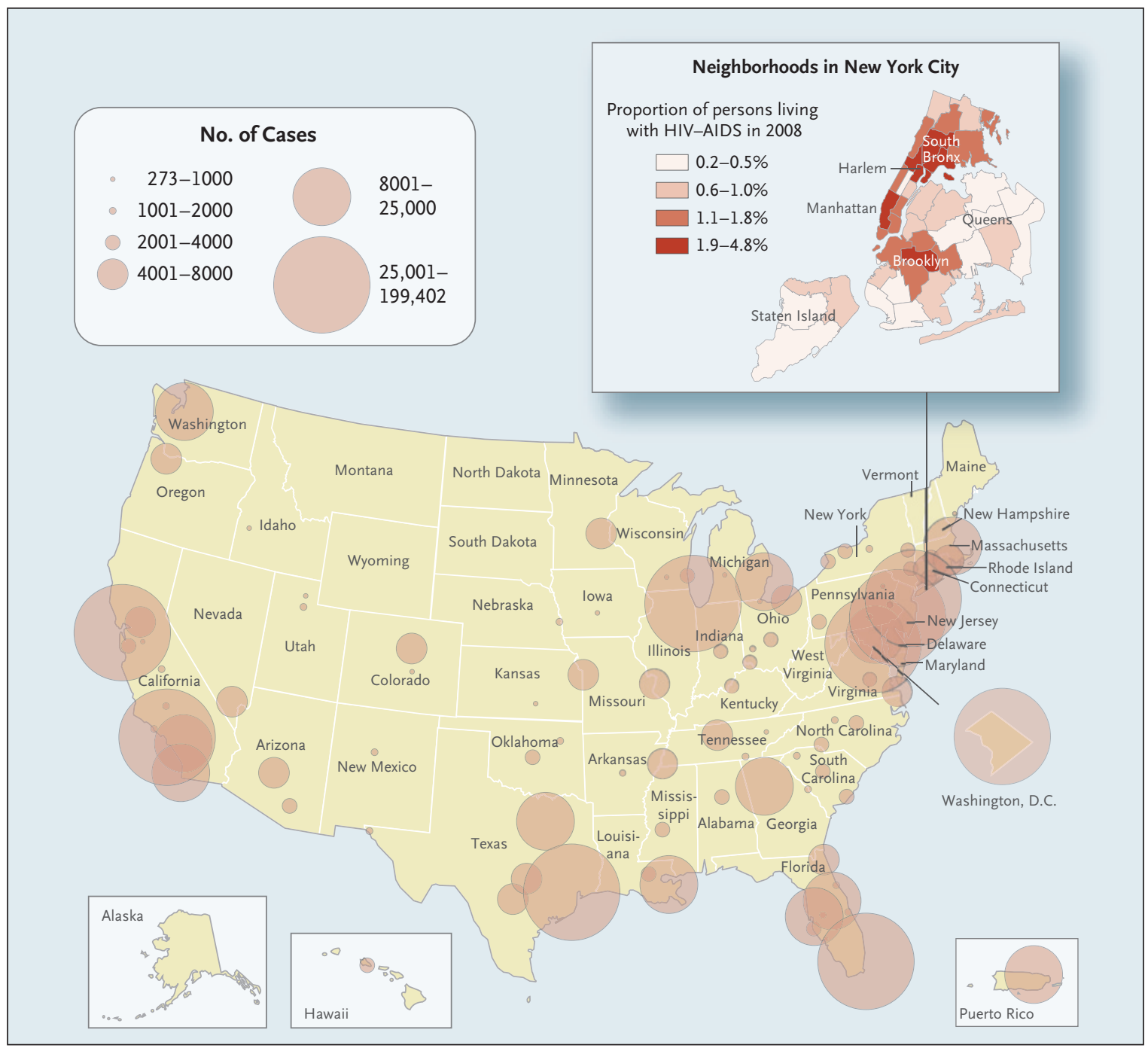

Numbers of Reported AIDS Cases According to Metropolitan Statistical Area of Residence, Cumulative through 2007.

Only metropolitan statistical areas with a population of more than 500,000 were included. Data are from the HIV/AIDS Surveillance Report, 2007, Centers for Disease Control and Prevention. The inset shows the proportion of persons living with HIV-AIDS in New York City. Data are from the New York City Department of Health and Mental Hygiene, Epidemiology and Field Services Program, October 2009.

in making decisions about sexual risk taking.

Most glaringly, HIV disproportionately affects poor black Americans who have substandard education, unstable housing, and limited social mobility. This confluence of factors may result in high rates of incarceration, which threaten a community's social fabric. Such vulnerable populations must be engaged in research, program development, and interven- tions that are culturally relevant and address the socioeconomic milieu in which HIV transmission occurs.

Preventive interventions must be rooted in science, not driven by ideological concerns. Homophobia may have impeded the development of sexually appropriate prevention studies among men who have sex with men. Reluctance to fund studies of needle exchange or conditional cash transfer (providing financial incentives for healthy behavior) or to support work in high-risk venues, such as bathhouses, has hampered progress. Cash transfer has proved effective in achieving desirable health outcomes, including weight control, smoking cessation, and decreased use of crystal methamphetamine, but until recently it was not being studied for use in HIV prevention in the United States. 
What will it take to control the U.S. HIV epidemic? First, there is an urgent need to acknowledge that HIV remains a major health threat in the United States. Second, concerted effort and substantial resource investment especially in innovative and courageous approaches - are necessary. Focused studies of the sociocultural dynamics that facilitate transmission are needed, as well as large studies assessing the effectiveness of multidimensional interventions, including behavioral, biomedical, and structural components. Disenfranchised communities must be engaged as partners in such efforts, along with new researchers drawn from the affected populations, if the nuances of local epidemics are to be addressed. The time has come to confront this largely forgotten and hidden epidemic.

Disclosure forms provided by the authors are available with the full text of this article at NEJM.org.

From the International Center for AIDS Care and Treatment Programs, Mailman School of Public Health, and the Department of Medicine, Harlem Hospital and College of Physicians and Surgeons, Columbia University, New York (W.M.E.-S.); the Fenway Institute at Fenway Health, Boston, and the Departments of Medicine and Community Health, Brown University, Providence, RI (K.H.M.); and the Department of Medicine, University of Medicine and Dentistry of New Jersey, Newark (S.L.H.).

This article (10.1056/NEJMp1000069) was published on February 10, 2010, at NEJM.org.

1. District of Columbia HIV/AIDS epidemiology update 2008. Washington, DC: Gov- ernment of the District of Columbia, Department of Health, HIV/AIDS Administration; 2008. (Accessed February 8, 2010, at http:// dchealth.dc.gov/DOH/frames.asp?doc=/ doh/lib/doh/pdf/dc_hiv-aids_2008_ updatereport.pdf.)

2. Nguyen TQ, Gwynn RC, Kellerman SE, et al. Population prevalence of reported and unreported HIV and related behaviors among household adult population in New York City, 2004. AIDS 2008;22:281-7.

3. HIV prevalence among selected populations: high-risk populations. Atlanta: Centers for Disease Control and Prevention, 2007. (Accessed February 8, 2010, at http://www .cdc.gov/hiv/topics/testing/resources/ reports/hiv_prevalence/high-risk.htm.)

4. Hall HI, Song R, Rhodes P, et al. Estimation of HIV incidence in the United States. JAMA 2008;300:520-9.

5. Millett GA, Flores SA, Peterson JL, Bakeman R. Explaining disparities in HIV infection among black and white men who have sex with men: a meta-analysis of HIV risk behaviors. AIDS 2007;21:2083-91.

Copyright () 2010 Massachusetts Medical Society.

\title{
Five Next Steps for a New National Program for Comparative-Effectiveness Research
}

\author{
Jordan M. VanLare, A.B., Patrick H. Conway, M.D., and Harold C. Sox, M.D.
}

The American Recovery and Reinvestment Act appropriated $\$ 1.1$ billion to fund comparative-effectiveness research (CER) - unprecedented generosity for a program for evaluating health care practices. The legislation established the Federal Coordinating Council for Comparative Effectiveness Research and charged it with advising the secretary of health and human services on the allocation of CER funds. It also mandated an Institute of Medicine (IOM) study to recommend initial national priorities for CER. Both the Federal Coordinating Council and the IOM reported to Congress on June 30, 2009.

Both organizations solicited input from stakeholders. The IOM committee issued an open solicitation asking the public to nomi- nate research topics. It received 1546 nominations, which it narrowed to 100 highest-priority research questions. The Federal Coordinating Council hosted three public listening sessions to identify priorities and posted drafts of its work on its Web site for public comment. By establishing a national CER agenda with input and support from diverse stakeholders, the two reports moved the United States closer to creating a sustained national CER program. ${ }^{1,2}$

Both reports recognized the need for a robust CER enterprise. The IOM made 10 recommendations for its development (see box). The Federal Coordinating Council's report included a definition of CER, a strategic framework, priority-setting criteria, and rec- ommendations for investing the $\$ 400$ million that Congress allocated to the Department of Health and Human Services for CER. Both reports recommended creating CER data networks and conducting research on practitioners' adoption of changes based on CER findings. ${ }^{3}$

Federal agencies and Congress appear willing to implement these recommendations. The National Institutes of Health, the Agency for Healthcare Research and Quality (AHRQ), and the secretary of health and human services have begun to allocate their Recovery Act funds and coordinate their efforts. The AHRQ has requested proposals for studying the IOM's high-priority research questions that fit within its own priorities,${ }^{4}$ and the secretary of health 